\title{
Characteristics of scattered electron beams shaped with a multileaf collimator $^{\text {a) }}$
}

\author{
Jean M. Moran ${ }^{\text {b) }}$ and Mary K. Martel \\ Department of Radiation Oncology, University of Michigan Medical Center, Ann Arbor, Michigan 48109 \\ laï A. D. Bruinvis \\ Department of Radiotherapy, The Netherlands Cancer Institute, Plesmanlaan 121, 1066 CX, Amsterdam, \\ The Netherlands \\ Benedick A. Fraass \\ Department of Radiation Oncology, University of Michigan Medical Center, Ann Arbor, Michigan 48109
}

(Received 27 March 1996; accepted for publication 30 June 1997)

\begin{abstract}
Characteristics of dual-foil scattered electron beams shaped with a multileaf collimator (MLC) (instead of an applicator system) were studied. The electron beams, with energies between 10 and $25 \mathrm{MeV}$, were produced by a racetrack microtron using a dual-foil scattering system. For a range of field sizes, depth dose curves, profiles, penumbra width, angular spread in air, and effective and virtual source positions were compared. Measurements were made when the MLC alone provided collimation and when an applicator provided collimation. Identical penumbra widths were obtained at a source-to-surface distance of $85 \mathrm{~cm}$ for the MLC and $110 \mathrm{~cm}$ for the applicator. The MLCshaped beams had characteristics similar to other machines which use trimmers or applicators to collimate scanned or scattered electron beams. Values of the effective source position and the angular spread parameter for the MLC beams were similar to those of the dual-foil scattered beams of the Varian Clinac 2100CD and the scanned beams of the Sagittaire linear accelerators. A model, based on Fermi-Eyges multiple scattering theory, was adapted and applied successfully to predict penumbra width as a function of collimator-surface distance. (C) 1997 American Association of
\end{abstract} Physicists in Medicine. [S0094-2405(97)02209-8]

Key words: racetrack microtron, electron beams, radiation therapy, multileaf collimator, penumbra width

\section{INTRODUCTION}

To date, most electron fields have been collimated by shaped lead-alloy cutouts placed inside electron applicators. With some accelerator systems, however, it is also possible to use the multileaf collimator (MLC) for electron field shaping. MLC-shaped electron beams could permit computercontrolled setup of new types of treatment fields. Complex field shapes could be treated quickly and easily without the need to enter the treatment room to set up heavy applicators or to change inserts in applicators. In preparation for clinical implementation of MLC-shaped electron beams, this work analyzes the use of a computer-controlled double-focused MLC alone (in place of applicators) for electron beam shaping with dual-foil scattered electron beams with energies from 10 to $25 \mathrm{MeV}$.

Most research with MLCs has focused on its use with photon beams, ${ }^{1-6}$ although some work has been done with multivane collimator systems for electron arc therapy. ${ }^{7-9}$ Brahme $^{10}$ first described the use of MLCs with electrons in place of applicators for scanned electron beams. Karlsson et al. ${ }^{11}$ recently reported the characteristics of high energy (up to $50 \mathrm{MeV}$ ) scanned electron beams. Field matching for electron and photon fields with a multileaf collimator has been reported on by Karlsson and Zackrisson. ${ }^{12}$

To take full advantage of the potential of multileafcollimated electron beams for conformal treatments, we must understand how the dose distributions change with MLC setting. These issues have been investigated for electron beams with applicators or trimmer bars. ${ }^{13-18}$ Klein et al. ${ }^{19}$ studied the collimation of dual-foil scattered electron beams with the Varian MLC to evaluate the potential use of this MLC with electrons.

All previous work on the racetrack microtron has addressed the scanned beam gantry. Karlsson et al. ${ }^{11}$ have presented the electron characteristics of a single gantry $50 \mathrm{MeV}$ racetrack microtron, describing the elementary beam, scanning pattern, and angular spread of the beam at the collimator level. Masterson et al. ${ }^{20}$ have described the reliability testing of the $50 \mathrm{MeV}$ racetrack microtron as well as the characteristics of the scanned electron beams. ${ }^{21}$

This work describes experiments which directly compare use of a MLC to the use of conventional applicators for foil-scattered electron beams. Comparisons between the two collimation methods are based on measurements of relative depth dose curves and profiles, analysis of penumbra width for square and rectangular fields, angular spread in air, and determination of effective and virtual source positions.

\section{MATERIALS AND METHOD}

\section{A. Racetrack microtron}

Most of this work was performed on a racetrack microtron (MM50 Racetrack Microtron System, Scanditronix 


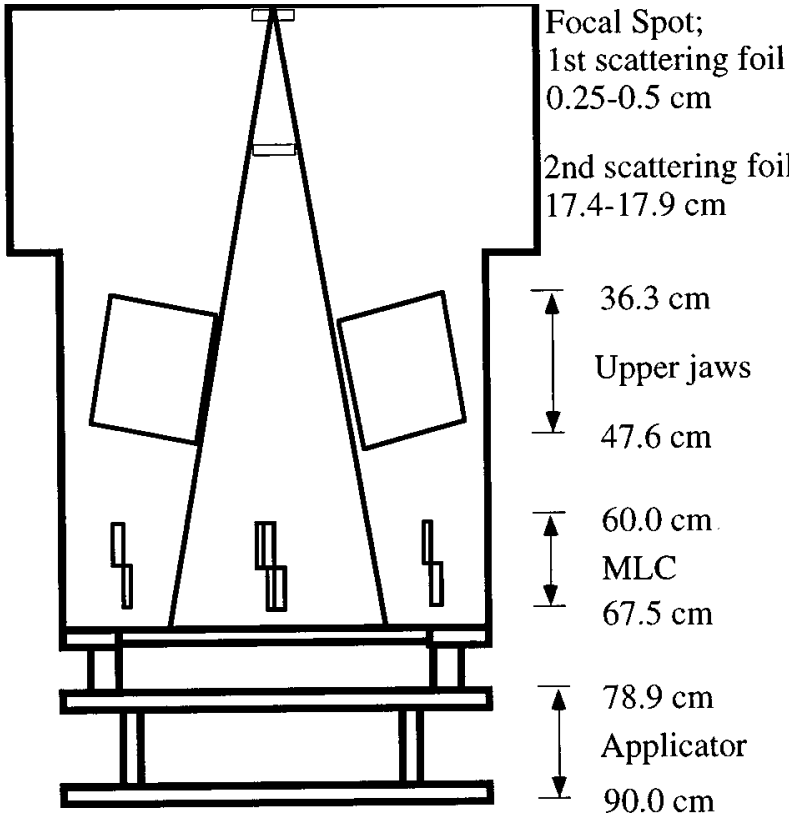

Fig. 1. Diagram of experimental setup. Distances are measured from a point $100 \mathrm{~cm}$ upstream of isocenter. Distances for the upper jaws and MLC are measured with the collimators closed.

AB, Uppsala, Sweden) with two different treatment gantries. Brahme et al. ${ }^{22}$ described the design of the Racetrack Microtron as well as the advantages of using scanning photon and electron beams. Electrons are accelerated in $5 \mathrm{MeV}$ steps with treatment energies ranging from 10 to $50 \mathrm{MeV}$. In our system, the beam can be transmitted from the accelerator room to either of two treatment gantries using a switching magnet and beam transport system. The beams studied in this work are scattered with a dual-foil system through a head open to air, with energies ranging from $10-25 \mathrm{MeV}$.

A diagram of the treatment head (Fig. 1) depicts the location of the beam forming structures. The first scattering foil is made of gold (gold and brass for $10 \mathrm{MeV}$ ) and ranges in thickness from 0.025 to $0.1 \mathrm{~mm}$ depending on the energy. The second foil is composed of multiple disks of varying radii and thickness which flatten the beam to the desired specifications. The same secondary scattering foil is used for 10 and $15 \mathrm{MeV}$. Different secondary foils are used for both 20 and $25 \mathrm{MeV}$ electrons. The $25 \mathrm{MeV}$ scattering foil was designed to preserve the deep penetration of the beam at the expense of flatness for field sizes greater than $20 \times 20 \mathrm{~cm}$.

Collimation of the scattered beam is provided by the primary collimator, upper photon jaws, the MLC, and the applicator (when used). The MLC consists of 32 pairs of double-focused tungsten leaves, each having a projected width of $1.25 \mathrm{~cm}$ at isocenter. ${ }^{11}$ It takes the place of the lower jaws in the collimator assembly and has a maximum field size of $31 \times 40 \mathrm{~cm}$ at $100 \mathrm{~cm}$ from the focal spot. The leaves move in an arc, following divergence, and are trapezoid shaped to minimize penumbra. The proximal point of the leaves from the source is at a distance of $67.5 \mathrm{~cm}$. As part of this investigation, the closest treatment distance for patient comfort was chosen to be between 85 and $90 \mathrm{~cm}$. Additional measurements were taken at other SSDs as well.

The applicator is composed of aluminum and has no closed walls. The square and rectangular inserts into the applicators are made of lead, while shaped field inserts are made of cerrobend $(2 \mathrm{~cm}$ thick). Four applicators provide field sizes of $10 \mathrm{~cm} \times 10 \mathrm{~cm}, 15 \mathrm{~cm} \times 15 \mathrm{~cm}, 20 \mathrm{~cm} \times 20 \mathrm{~cm}$, and $25 \mathrm{~cm} \times 30 \mathrm{~cm}$. When an applicator is used clinically, the upper jaws are fully retracted and the MLC is set to a field size $5 \mathrm{~cm}$ greater than the applicator field size (defined at isocenter), for all sizes and energies. The upper jaws were fully retracted for all experiments in this work. The applicator (when used) is mounted on the collimator housing $(4 \mathrm{~cm}$ below the MLC) and its end is located at a distance of $90 \mathrm{~cm}$ from the source (Fig. 1). The standard treatment distance for the applicators is at $100 \mathrm{~cm}$ from the source.

\section{B. Measurements}

Dosimetric measurements were made with ion chambers and diodes in a water phantom (WP-700, Wellhofer Dosimetrie, Germany). Relative depth dose curves and profiles were measured with a $p$-type silicon diode detector (Electron Diodes, Scanditronix AB, Uppsala, Sweden) with a chip diameter of $2.5 \mathrm{~mm}$. Accuracy of detector setup in the water tank was $+/-0.5 \mathrm{~mm}$ in $x$ and $y$ directions and $+/$ $-1 \mathrm{~mm}$ in the $z$ (depth) direction. Profile measurement depths were determined from the depth dose curves. Depths included $0.5 \mathrm{~cm}, \mathrm{R}_{100}, \mathrm{R}_{90}, \mathrm{R}_{80}, \mathrm{R}_{50}, \mathrm{R}_{20}, \mathrm{R}_{p}$, and $\mathrm{R}_{p}+5$ $\mathrm{cm} .{ }^{23}$ Field sizes were defined at the standard treatment distance of $85 \mathrm{~cm}$ for the MLC and $100 \mathrm{~cm}$ for the applicators. The field sizes studied were $5 \mathrm{~cm} \times 5 \mathrm{~cm}, 10 \mathrm{~cm} \times 10 \mathrm{~cm}$, $15 \mathrm{~cm} \times 15 \mathrm{~cm}, \quad 20 \mathrm{~cm} \times 20 \mathrm{~cm}$, and $25 \mathrm{~cm} \times 30 \mathrm{~cm}$. The complete set of curves was measured for 85,90 , and $100 \mathrm{~cm}$ SSD when the MLC was used, and at 100 and 110 SSD for the applicator.

$\sigma_{\theta_{x}}$ is a measure of the beam's angular spread and is related to the penumbra. To determine its value, profiles were measured with silicon diodes in air for a $20 \mathrm{~cm} \times 20 \mathrm{~cm}$ field at distances of 80 to $110 \mathrm{~cm}$ from the focal spot for the MLC and 95 to $120 \mathrm{~cm}$ for the applicator. The initial angular divergence, $\sigma_{\theta_{x}}$, was determined by the following relations: ${ }^{24}$

$$
\sigma_{\theta_{x}}=0.391 * \text { slope }_{90 \%-10 \%}
$$

or

$$
\sigma_{\theta_{x}}=0.595 * \text { slope }_{80 \%-20 \%},
$$

where the slope represents the linear fit to the $90 \%-10 \%$ or $80 \%-20 \%$ penumbra widths plotted against the SSD or the distance below the collimator. The value of $\sigma_{\theta_{x}}$ was determined for both the MLC and applicator for all electron beams. In addition, measurements were made for all energies of the Varian Clinac 2100 CD (Clinac 2100 CD, Varian Associates, Inc., Palo Alto, CA) with the $20 \mathrm{~cm} \times 20 \mathrm{~cm}$ applicator for comparison. 
The profiles in air were also used to determine the virtual source position (VSD, the point from which the beam appears to be diverging), by a back projection of the width between the $50 \%$ points of profiles. ${ }^{25}$

The effective source position (ESD) for inverse square law output correction was determined using the method of Khan et al. ${ }^{26}$ A $0.69 \mathrm{cc}$ ion chamber (Farmer model 2571, Nuclear Enterprises Ltd., England) was set at $2.5 \mathrm{~cm}$ depth in solid water while the distance from the collimator to the phantom surface was varied for a number of field sizes. The effective source position was determined relative to $85 \mathrm{~cm}$ for the MLC and to $100 \mathrm{~cm}$ for the applicators. The ESD was determined by a linear fit to the data.

\section{Analysis methods}

It is known that the penumbra width is dependent upon energy, depth, source-to-collimator distance, source-tosurface distance, and whether or not the beam is scattered or scanned. In addition, it depends on scatter effects due to foils, collimation, air, and the phantom. Based on FermiEyges theory, the penumbra width is related to the pencil beam spread in air and in water as follows: ${ }^{24}$

$$
W_{80 \%-20 \%}(z)=1.68 *\left[\sigma_{\theta_{x}}^{2}(d+z)^{2}+\sigma_{\text {water }}^{2}(z)\right]^{1 / 2},
$$

where $d$ is the gap from the collimator to the phantom surface, $z$ is the depth in water, $\sigma_{\theta_{x}}$ is the angular spread in air at the level of the collimator, and $\sigma_{\text {water }}$ is the pencil beam lateral spread in water. Values for $\sigma_{\text {water }}$ were calculated using the approximation by Hogstrom. ${ }^{24}$ Because the $\sigma_{\text {water }}$ calculation only accounts for small angle scattering, a correction factor is applied, the Fermi multiple Coulomb scatter $(\mathrm{FMCS})^{24}$ correction term, to correctly predict the scattering in water. Values of $\sigma_{\theta_{x}}$ and FMCS were determined from a best fit over the range of measured SSDs to penumbra values at a depth of dose maximum. Results were compared to the measured values.

\section{RESULTS}

Differences between the depth doses measured with the MLC and with the applicator were very small in most situations, and the depth doses were the same for fields of $10 \mathrm{~cm} \times 10 \mathrm{~cm}$ and greater. Depth dose curves for MLC and applicator beams are plotted in Fig. 2 for $5 \mathrm{~cm} \times 5 \mathrm{~cm}$ and $10 \mathrm{~cm} \times 10 \mathrm{~cm}$ fields for 10 and $25 \mathrm{MeV}$. Depth dose curves for the applicator were measured at $100 \mathrm{~cm}$ SSD. Depth dose curves for the MLC were measured at $85 \mathrm{~cm} \mathrm{SSD} \mathrm{and} \mathrm{then}$ corrected for inverse square law to $100 \mathrm{~cm}$. The surface dose is approximately $82 \%$ of the maximum for $10 \mathrm{MeV}$ and $90 \%$ for $25 \mathrm{MeV}$. Dose maximum occurs at $2.3 \mathrm{~cm}$ for $10 \mathrm{MeV}$ and $3 \mathrm{~cm}$ for 15,20 , and $25 \mathrm{MeV}$. There is a slight difference between the MLC and applicator depth dose curves at 25 $\mathrm{MeV}$ for the $5 \mathrm{~cm} \times 5 \mathrm{~cm}$ field size which is most likely due to additional low energy electrons scattered by the insert.

Profiles at a depth of $0.5 \mathrm{~cm}$ for $10 \mathrm{MeV}$ electrons are shown in Fig. 3(a) for $5 \mathrm{~cm} \times 5 \mathrm{~cm}$ and $10 \mathrm{~cm} \times 10 \mathrm{~cm}$ fields for the applicator at $100 \mathrm{~cm}(10 \mathrm{~cm}$ air gap) and the MLC at

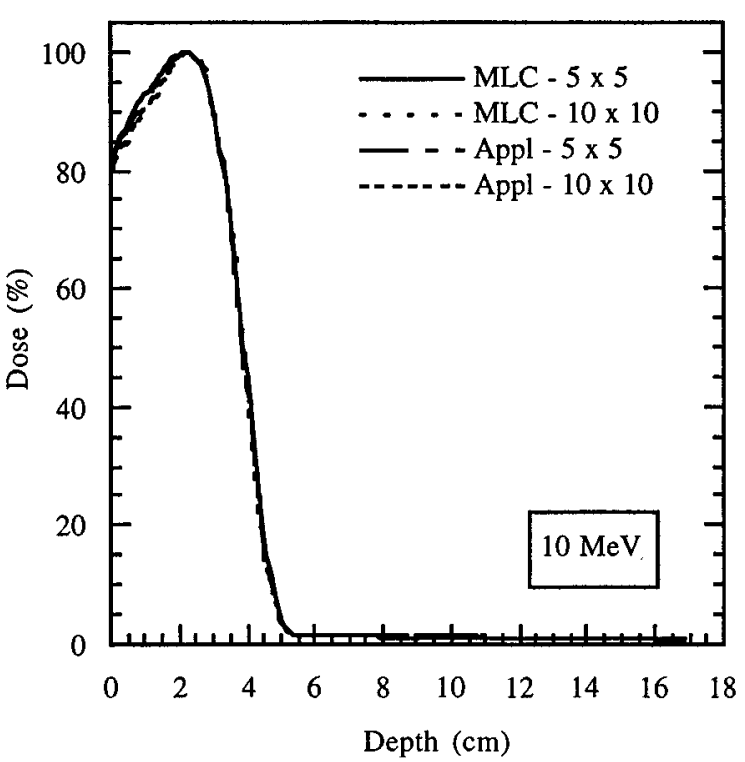

(a)

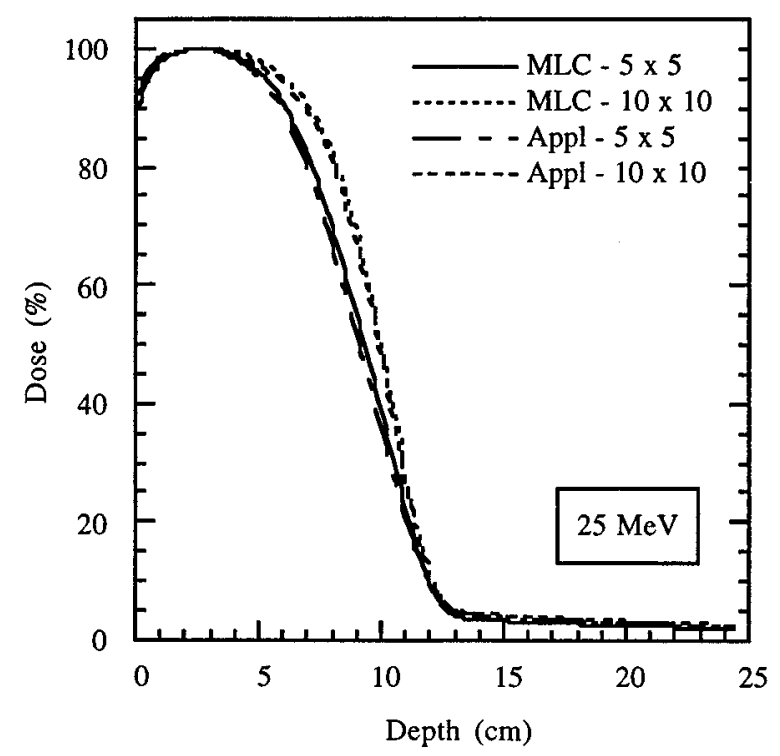

(b)

FIG. 2. Depth dose curves for dual-foil scattered electron beams (G25) for $5 \times 5 \mathrm{~cm}$ and $10 \times 10 \mathrm{~cm}$ field collimated by the MLC or applicator for (a) $10 \mathrm{MeV}$ and (b) $25 \mathrm{MeV}$. The MLC curves were measured at $85 \mathrm{~cm} \mathrm{SSD}$ and corrected for inverse square law to $100 \mathrm{~cm} \mathrm{SSD}$

$85 \mathrm{~cm}(17.5 \mathrm{~cm}$ air gap). As expected due to the increased collimator-surface distance, the penumbra is larger for the MLC field. This results in a profile for the $5 \mathrm{~cm} \times 5 \mathrm{~cm}$ field which has no flat region. Figure 3(b) shows the profiles for the same field sizes for $25 \mathrm{MeV}$ at a depth of $3.0 \mathrm{~cm}$. For this energy, agreement between the MLC and applicator fields is better for all field sizes. A Bremsstrahlung dose level of less 


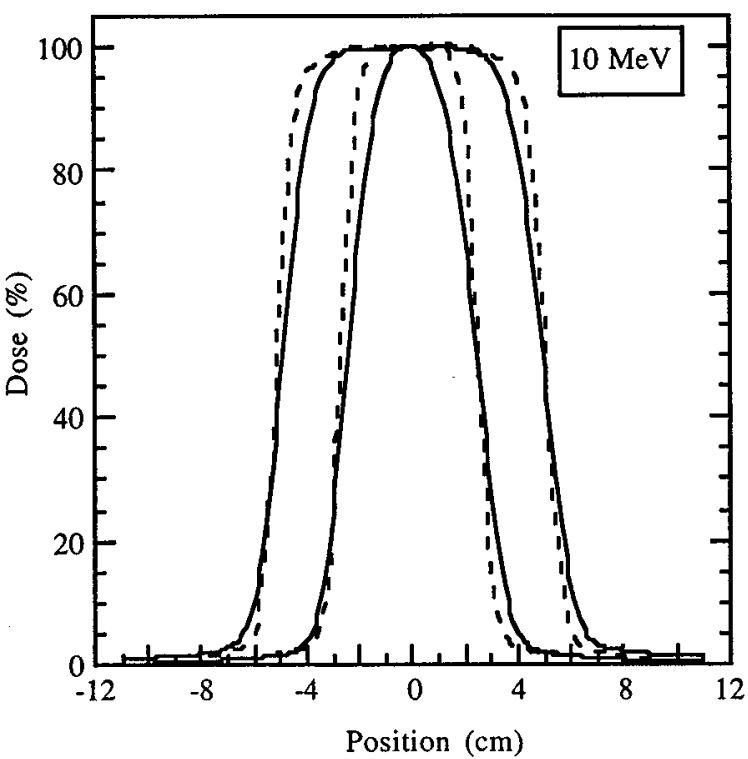

(a)

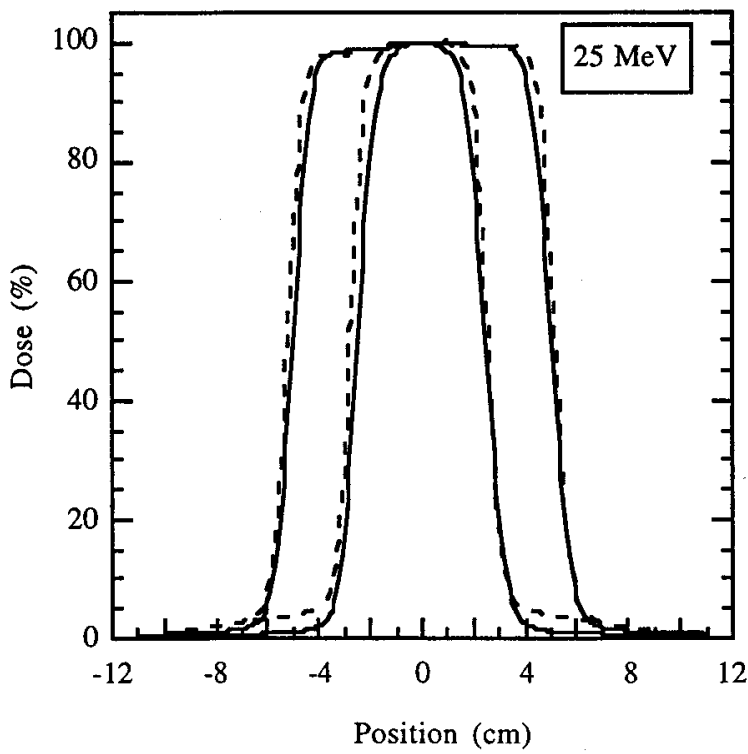

(b)

FIG. 3. Profiles for $5 \mathrm{~cm} \times 5 \mathrm{~cm}$ and $10 \mathrm{~cm} \times 10 \mathrm{~cm}$ fields (a) at a depth of 0.5 $\mathrm{cm}$ for $10 \mathrm{MeV}$ electrons and (b) at a depth of $3.0 \mathrm{~cm}$ for $25 \mathrm{MeV}$ electrons collimated by MLC (solid lines) or applicator (dashed lines). The MLC curves were measured at $85 \mathrm{~cm}$ SSD and the applicator curves were measured at $100 \mathrm{~cm} \mathrm{SSD.}$

than $5 \%$ originating from the cerrobend insert can be seen for the $5 \mathrm{~cm} \times 5 \mathrm{~cm}$ field.

Flatness was determined from profiles at a depth of nominal dose maximum for which the area $2 \mathrm{~cm}$ within the geometric field edge was examined. ${ }^{23}$ For a $20 \mathrm{~cm} \times 20 \mathrm{~cm}$ field at $100 \mathrm{~cm} \mathrm{SSD}$, the flatness for the applicator profiles was better than $2 \%$ for $10 \mathrm{MeV}$ and better than $1 \%$ for $25 \mathrm{MeV}$. For the same field size at $85 \mathrm{~cm} \mathrm{SSD}$, the flatness for the MLC curves was within $4.0 \%$ for $10 \mathrm{MeV}$ and within 3\% for $25 \mathrm{MeV}$.

Penumbra values are shown in Fig. 4(a) for $10 \mathrm{MeV}$ at 2.3 $\mathrm{cm}$ and 4(b) for $25 \mathrm{MeV}$ at $3.0 \mathrm{~cm}$. The penumbra widths are plotted versus the distance from the collimator to the phantom for a number of field sizes. There was a $2-3 \mathrm{~mm}$ increase in penumbra for the MLC fields when compared to the applicator fields at identical distances from the collimators. The SSD at which the MLC penumbra widths was the same as the applicator penumbra width at $110 \mathrm{~cm}$ SSD was found from Fig. 4 to be $85 \mathrm{~cm} \mathrm{SSD} \mathrm{for} \mathrm{both} 10$ and $25 \mathrm{MeV}$.

The $\sigma_{\theta_{x}}$ values obtained from the in-air measurements of the $80 \%-20 \%$ penumbra width are shown in Table I for 10 and $25 \mathrm{MeV}$. The spread in air was slightly greater for the MLC than for the applicator. Comparison to other values for other machines is shown later. Equation (3) was used to calculate the penumbra widths once the parameter values were determined from a fit to the penumbra widths at a depth of dose maximum. Figure 5 shows the calculated penumbra values and those measured for a $20 \times 20 \mathrm{~cm}$ field for both the applicator and the MLC for (a) $10 \mathrm{MeV}$ and (b) $25 \mathrm{MeV}$.

Virtual (full width at half-maximum) and effective (output) source positions are shown in Table II as the distance downstream from the focal spot for 10 and $25 \mathrm{MeV}$. The virtual source positions are approximately the same for both the MLC and applicator. The virtual source position for 10 $\mathrm{MeV}$ is further downstream due to increased scattering in air. For the $20 \mathrm{~cm} \times 20 \mathrm{~cm}$ field, the effective source position was located up to $5 \mathrm{~cm}$ downstream from the virtual source position, depending on the energy.

The effective source positions were determined for a number of sizes of both MLC and applicator fields and are given in Table III. Values were similar for the MLC and applicator fields.

\section{DISCUSSION}

In Fig. 4, the penumbra variation with field size and with distance from the collimator is seen. For $10 \mathrm{MeV}$, the penumbra for the $5 \times 5 \mathrm{~cm}$ field size was approximately $1 \mathrm{~mm}$ less than for the $10 \times 10$ and $20 \times 20 \mathrm{~cm}$ field sizes (probably due to lack of lateral scatter equilibrium). This was seen for both the applicator and the MLC. For fields greater than 5 $\times 5 \mathrm{~cm}$, the penumbra values were similar. For $25 \mathrm{MeV}$, there was approximately a $2 \mathrm{~mm}$ difference in penumbra for the $20 \times 20 \mathrm{~cm}$ field compared to the $5 \times 5 \mathrm{~cm}$ and 10 $\times 10 \mathrm{~cm}$. This effect is not seen for the applicator field. It is not clear what caused this difference.

A relationship based on Fermi-Eyges theory was used to predict penumbra values for both the applicator and MLC. Based on geometry, the penumbra is expected to be greater for the MLC than for the applicator at the same distance from the collimators because of the shorter source-tocollimator distance (SCD). This dependence on the SCD explained most of the difference in penumbra between the applicator and MLC. This is shown in Fig. 4, where larger 


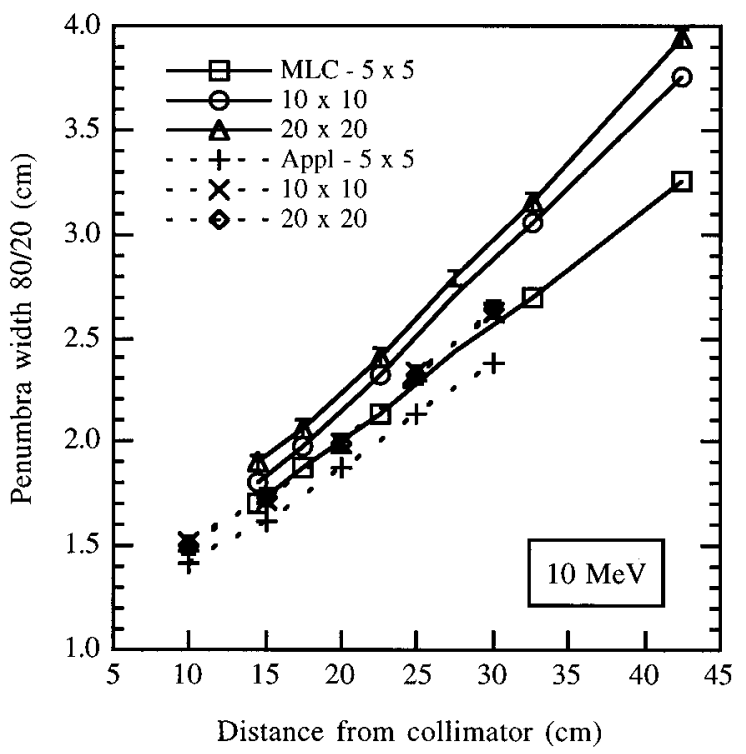

(a)

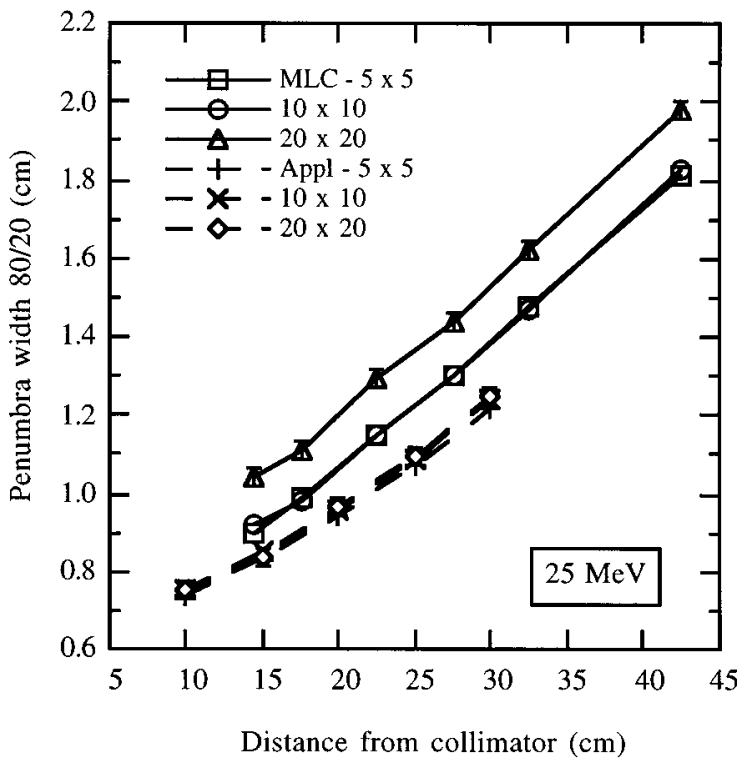

(b)

FIG. 4. Penumbra measurements for $5 \mathrm{~cm} \times 5 \mathrm{~cm}, 10 \mathrm{~cm} \times 10 \mathrm{~cm}$, and $20 \mathrm{~cm} \times 20 \mathrm{~cm}$ for collimation by MLC and applicator for (a) $10 \mathrm{MeV}$ at 2.3 $\mathrm{cm}$ and (b) $25 \mathrm{MeV}$ at $3.0 \mathrm{~cm}$ in water. Error bars are shown for the $20 \mathrm{~cm} \times 20 \mathrm{~cm}$ fields and represent one standard deviation in the measurements.

penumbra values are predicted for the MLC when compared to the applicator at identical distances from the collimators.

The penumbra widths were calculated using Eq. (3), where $\sigma_{\theta_{x}}$ and FMCS were derived from a fit to the penumbra widths at a depth of dose maximum. The results were compared to the measured values for the $20 \times 20 \mathrm{~cm}$ field as shown in Fig. 5, for the MLC and applicator. For both the
TABLE I. Values of $\sigma_{\theta}(80 \%-20 \%)$ (in radians) and FMCS for applicator and MLC collimated electrons beams based on a $20 \mathrm{~cm} \times 20 \mathrm{~cm}$ field. Results were derived from in air measurements and from measurements at a depth of dose maximum to predict penumbra widths. Values for the Fermi multiple Coulomb scatter term are also shown. Error (shown as \pm ) is for 1 standard deviation and is in radians.

\begin{tabular}{clccc}
\hline \hline $\begin{array}{c}\text { Energy } \\
(\mathrm{MeV})\end{array}$ & Collimator & $\sigma_{\theta_{x}}$ derived in air & $\sigma_{\theta_{x}}$ from fit & FMCS from fit \\
\hline 10 & MLC & $0.0484 \pm 0.0005$ & 0.050 & 1.63 \\
10 & Applicator & $0.0435 \pm 0.001$ & 0.043 & 1.63 \\
25 & MLC & $0.0216 \pm 0.0005$ & 0.025 & 1.43 \\
25 & Applicator & $0.0164 \pm 0.0009$ & 0.018 & 1.43 \\
\hline \hline
\end{tabular}

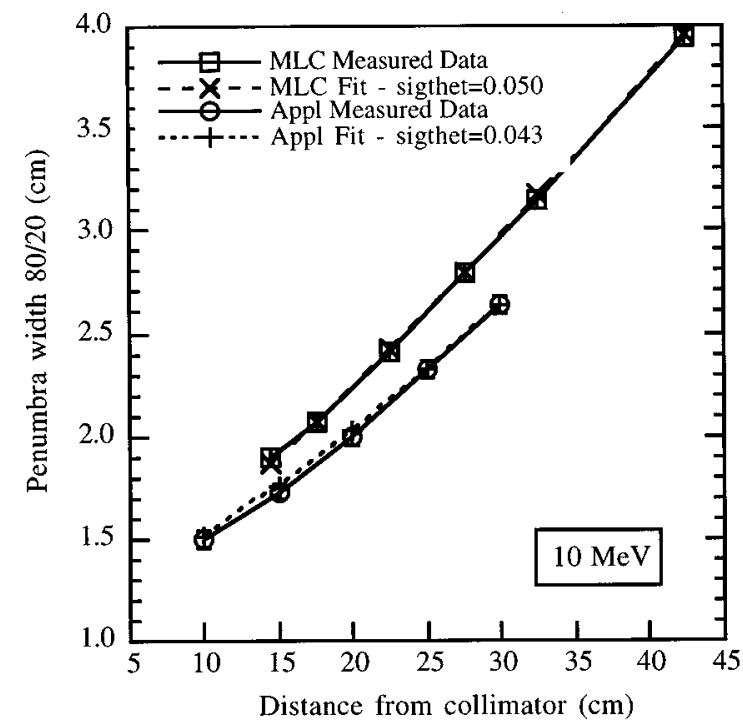

(a)

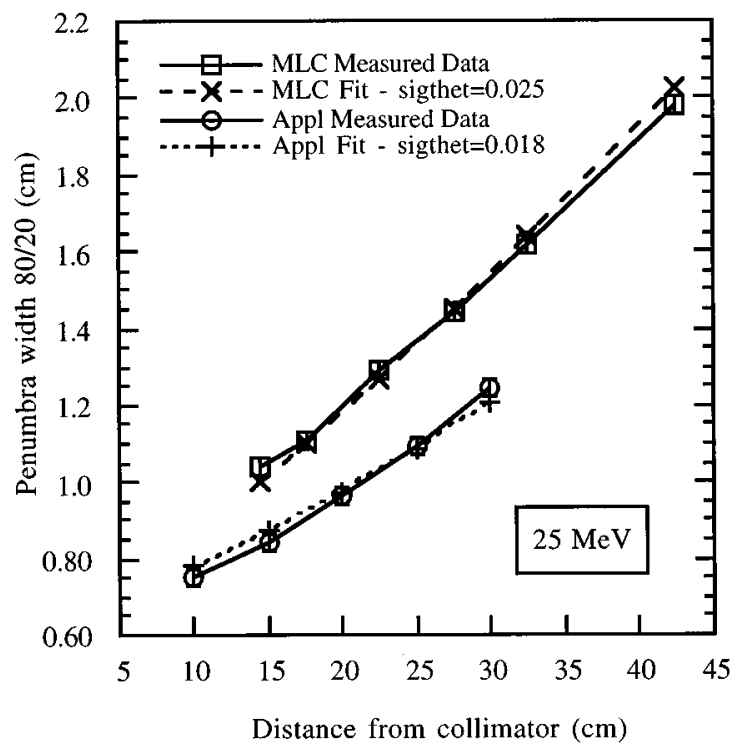

(b)

FIG. 5. A comparison of the pencil beam model and the $20 \times 20 \mathrm{~cm}$ penumbra data as a function of distance from the collimator for (a) $10 \mathrm{MeV}$ at 2.3 $\mathrm{cm}$ and (b) $25 \mathrm{MeV}$ at $3.0 \mathrm{~cm}$ in water. 
TABLE II. Source positions downstream from the focal spot for a $20 \mathrm{~cm} \times 20 \mathrm{~cm}$ field for both the MLC and the applicator (in $\mathrm{cm}$ ). Error (shown as \pm ) is for 1 standard deviation and is in $\mathrm{cm}$.

\begin{tabular}{lcccc}
\hline \hline & $10 \mathrm{MeV}$ & $10 \mathrm{MeV}$ & $25 \mathrm{MeV}$ & $25 \mathrm{MeV}$ \\
\hline Collimator & Effective & Virtual source & Effective & Virtual source \\
& source position & position & source position & position \\
MLC & $10.4 \pm 0.1$ & $9.1 \pm 0.4$ & $7.2 \pm 0.1$ & $2.4 \pm 1.5$ \\
Applicator & $10.7 \pm 0.4$ & $8.9 \pm 0.6$ & $7.6 \pm 0.3$ & $2.5 \pm 1.6$ \\
\hline \hline
\end{tabular}

MLC and applicator, values of $\sigma_{\theta_{x}}$ larger than those derived from in-air measurements are necessary to accurately calculate the penumbra over the range of distances from the collimator $(10-43 \mathrm{~cm})$ studied here. The in-air measured value of $\sigma_{\theta_{x}}$ seems to be inappropriate for predicting the penumbra. Results were evaluated over the SSDs of interest. Table I contains a summary of the values derived in air and determined from penumbra data at a depth of dose maximum for 10 and $25 \mathrm{MeV}$. Values of FMCS, derived from the fit, are also presented.

The value of $\sigma_{\theta_{x}}$ is related inversely to the SCD through the penumbra. As expected, the values determined from measurements were smaller for the applicator which has a larger SCD than for the MLC. A comparison of $\sigma_{\theta_{x}}(90 \%-$ $10 \%$ ) values obtained for similar energies with other treatment machines is plotted in Fig. 6. Values are shown for the scanned beams of the CGR-Sagittaire ${ }^{27}$ (with trimmers at $88.5 \mathrm{~cm}$ from the source) and the dual-foil scattered beams of the Scanditronix MM22 28 (with applicator end at $95 \mathrm{~cm}$ from the source), in addition to the values obtained in this work for the Varian $2100 \mathrm{CD}$ (with applicator end at $95 \mathrm{~cm}$

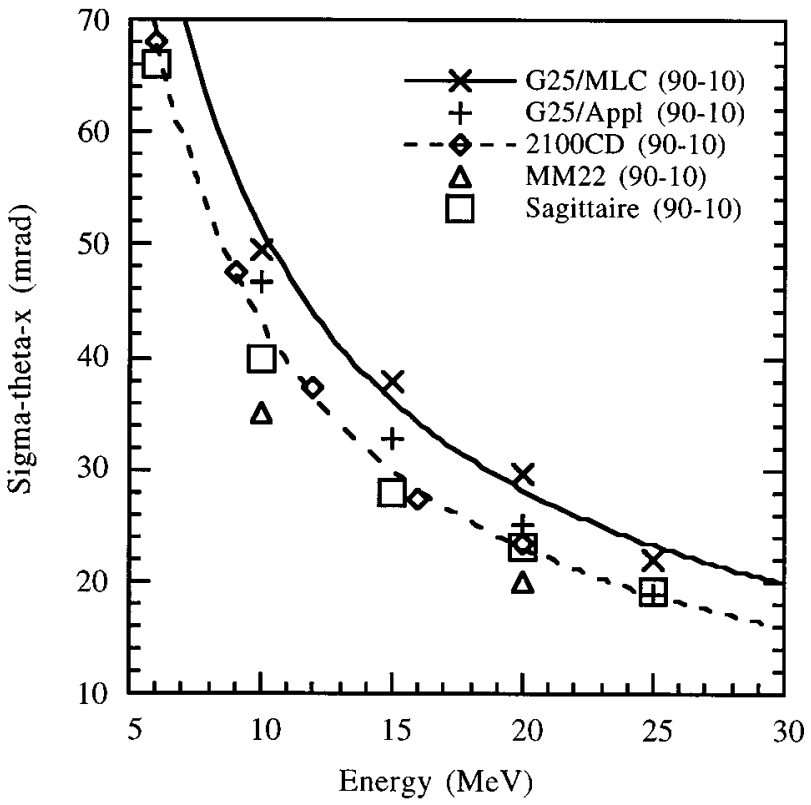

FIG. 6. Values of the angular spread parameter, $\sigma_{\theta_{x}},(90 \%-10 \%)$ for the microtron (measured), Clinac 2100CD (measured), and other machines (from Huizenga and Storchi, 1987). The lines for the G25/MLC and Clinac $2100 \mathrm{CD}$ beams are only to show the trend of these data points. from the source) and G25 (with MLC at $67.5 \mathrm{~cm}$ from the source and with applicator end at $90 \mathrm{~cm}$ from the source). The angular spread term is approximately 5 mradians greater for the MLC than with the applicator (due to the effect of the source-to-collimator distance). Measurements for the Clinac 2100 CD were slightly lower than those of G25. When compared to the scanned beams of the Sagittaire and the dual-foil scattered beams of the $2100 \mathrm{CD}$, the values obtained for the dual-foil scattered beams with the MLC are only slightly greater (especially for $25 \mathrm{MeV}$ ). Note that energies reported here are nominal energies.

Comparisons to other treatment machines can also be made for the effective source distance (ESD, based on the inverse square law). Figure 7(a) depicts the effective source position downstream from the target (towards the exit of the collimator) as a function of the field area for 9 and $10 \mathrm{MeV}$ electron beams of the G25/Appl, G25/MLC, Varian Clinac 2100 CD, Siemens Mevatron 80, ${ }^{25}$ AECL Therac 25, ${ }^{29}$ and Philips SL25. ${ }^{30}$ Figure 7(b) depicts the effective source position downstream from the target for 22 and $25 \mathrm{MeV}$ electron beams of the G25/Appl, G25/MLC, Therac $25,{ }^{29}$ Scanditronix MM22, ${ }^{28}$ and SL25. ${ }^{30}$ Nominal energies are reported. All machines except the G25/MLC, used trimmers or applicators. The effective source positions of the dual-foil scattered beams of the Mevatron 80 are within $5 \mathrm{~cm}$ of the focal spot. For the other dual-foil scattered beams, the effective source position is further from the focal spot, especially for smaller fields where lateral scatter equilibrium does not exist. For the MM50 dual-foil scattered beams collimated by either the applicator or MLC, the effective source positions are similar and between the first scattering foil (at $0.5 \mathrm{~cm}$ from the focal spot) and the second scattering foil (at 17.9 $\mathrm{cm})$. These values are slightly greater than those for the 9 $\mathrm{MeV}$ Clinac 2100CD beam for small field sizes. Values obtained for the 2100CD are similar to those reported by Roback et $a l .^{31}$ At larger field sizes, the values for the G25/ MLC are comparable to those obtained for other dual-foil scattered beams with applicators or trimmers such as the SL25, MM22, and Clinac 2100CD.

There are a number of constraints on the use of these data to estimate how other electron-MLC systems might work. On other types of machines, the penumbra for an applicator may not be linear as a function of distance from the collimator and it may vary more with field size, depending on the applicator design. For example, in many older machines, ap-

TABLE III. Position of the effective source downstream from the focal spot for both the MLC and applicators for 10 and $25 \mathrm{MeV}$ electron beams (in $\mathrm{cm})$. The $5 \mathrm{~cm} \times 5 \mathrm{~cm}$ field is an insert in the $10 \mathrm{~cm} \times 10 \mathrm{~cm}$ applicator. Error (shown as \pm ) is for 1 standard deviation and is in $\mathrm{cm}$.

\begin{tabular}{crrrr}
\hline \hline Field size $\left(\mathrm{cm}^{2}\right)$ & \multicolumn{2}{c}{$10 \mathrm{MeV}$} & \multicolumn{2}{c}{$25 \mathrm{MeV}$} \\
\hline & G25/MLC & G25/Appl & G25/MLC & G25/Appl \\
$5 \times 5$ & $36.7 \pm 1.1$ & $31.6 \pm 1.6$ & $11.5 \pm 0.4$ & $13.0 \pm 0.4$ \\
$10 \times 10$ & $15.8 \pm 0.4$ & $14.2 \pm 0.4$ & $7.0 \pm 0.1$ & $9.8 \pm 0.6$ \\
$15 \times 15$ & $11.3 \pm 0.2$ & $10.4 \pm 0.5$ & $7.3 \pm 0.1$ & $8.4 \pm 0.4$ \\
$20 \times 20$ & $10.4 \pm 0.1$ & $10.7 \pm 0.4$ & $7.2 \pm 0.1$ & $7.6 \pm 0.3$ \\
$25 \times 30$ & $11.5 \pm 0.3$ & $8.5 \pm 0.3$ & $7.3 \pm 0.1$ & $6.1 \pm 0.2$ \\
\hline \hline
\end{tabular}




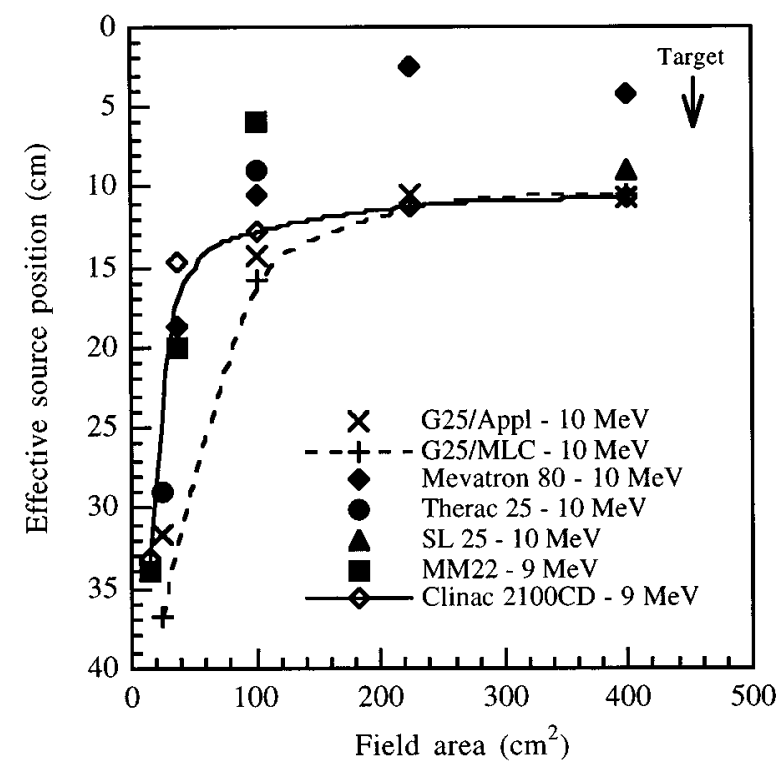

(a)

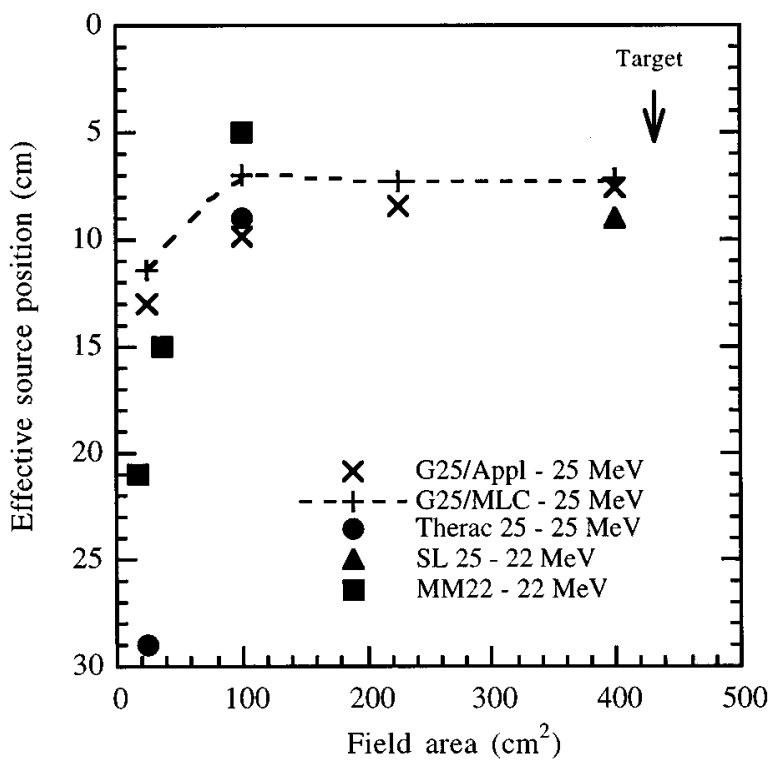

(b)

FIG. 7. Values of the effective source position downstream from the target as a function of the field area for a number of treatment machines (a) for 9 and $10 \mathrm{MeV}$ electron beams and (b) for 22 and $25 \mathrm{MeV}$ electron beams. The lines for the G25/MLC and Clinac 2100CD beams are only to show the trend of these data points.

plicators were designed to produce scattered electrons to improve the flatness of the electron beams. Newer designs, like the new design of dual-scattering foils for the Varian $2100 \mathrm{C},{ }^{32}$ decrease the amount of in-scatter which is used. Differences should not be as significant for machines that use trimmers, such as the Therac 25. For these machines, the trimmers' main function is to decrease the penumbra width.

The penumbra values are expected to be dependent on MLC design. The position of the MLC in the head, relative to its distance from the source and distance to the patient, affect the penumbra and angular spread. The shape of the edges (straight or curved) may affect the pathlength of electrons through individual leaves. Finally, the penumbra may also be affected by whether or not the MLC is doublefocused.

\section{CONCLUSIONS}

Based on a comparison of depth dose curves and profiles, beams defined by the MLC are similar to those using applicators for the square fields studied here. There is an increase of up to $3 \mathrm{~mm}$ in penumbra for the MLC when compared to the applicator for identical distances from the collimators for 10 and $25 \mathrm{MeV}$ electron beams. This is mainly due to the smaller source-to-collimator distance for the MLC compared to that of the applicator. From this work, it can be concluded that a double-focused MLC provides clinically adequate collimation for the dual-foil scattered beams of the racetrack microtron, when used at appropriate source-surface distances. An SSD of $85 \mathrm{~cm}$ provides collimation similar to that obtained with an applicator at $110 \mathrm{~cm}$ SSD (commonly used as an extended treatment distance). For this machine, we find that SSDs less than $85 \mathrm{~cm}$ are not considered clinically acceptable because of both the excessive height of the patient above the floor and the closeness of the patient to the collimator.

The measured values of the $\sigma_{\theta_{x}}$ parameter of the MDAH pencil beam model ${ }^{24}$ do not adequately predict the penumbra for the range of SSDs of interest with the MLC or applicator. This approximation ignores the contribution of scatter from sources such as the upper jaws, MLC, and/or applicator. In this work, it was shown that values of $\sigma_{\theta_{x}}$ derived from penumbra data at a depth of dose maximum were more appropriate for modeling. If this adaptation of the model is not sufficient for 3-D dose calculations, a more inclusive model such as the one developed by Brahme using the extended effective source ${ }^{33}$ may be necessary.

\section{ACKNOWLEDGMENTS}

This work was supported in part by NIH Grant No. P01CA59827 and by UICC/ICRETT Award No. 185/1995.

\footnotetext{
${ }^{a}$ Presented in part at the 1994 meeting of the American Association of Physicists in Medicine, Anaheim, California, July 1994.

${ }^{b)}$ Reprint request to: Jean M. Moran, Department of Radiation Oncology, University of Michigan Medical Center, Room B2C438, Box 0010, 1500 Medical Center Drive, Ann Arbor, Michigan 48109. Electronic-mail: jmmoran@umich.edu, Phone: (313) 763-9860, Fax: (313) 936-7859.

${ }^{1} \mathrm{~A}$. Brahme, "Optimal setting of multileaf collimators in stationary beam radiation therapy," Strahlentherapie und Oncologie. 164, 343-350 (1988).

${ }^{2} \mathrm{P}$. Biggs, J. Capalucci, and M. Russell, "Comparison of the penumbra between focused and non-divergent blocks - Implications for multileaf collimators," Med. Phys. 18, 753-758 (1991).

${ }^{3}$ A. L. Boyer, T. G. Ochran, C. E. Nyerick, T. J. Waldron, and C. J. Huntzinger, "Clinical dosimetry for implementation of a multileaf collimator," Med. Phys. 19, 1255-1261 (1992).
} 
${ }^{4}$ J. M. Galvin, A. R. Smith, R. D. Moeller, R. L. Goodman et al., “Evaluation of multileaf collimator design for a photon beam,', Int. J. Radiat. Oncol., Biol., Phys. 23, 789-801 (1992).

${ }^{5}$ J. M. Galvin, A. R. Smith, and B. Lally, 'CCharacterization of a multileaf collimator system,' Int. J. Radiat. Oncol., Biol., Phys. 25, 181-192 (1993).

${ }^{6}$ W. D. Powlis, A. R. Smith, E. Cheng, J. M. Galvin, F. Villari, P. Bolch, and M. Kligerman, "Initiation of multileaf conformal radiation therapy,", Int. J. Radiat. Oncol., Biol., Phys. 25, 171-179 (1993).

${ }^{7}$ D. D. Leavitt, L. M. Peacock, F. A. Gibbs, Jr., and J. R. Stewart, “Electron arc therapy: Physical measurement and treatment planning techniques,' Int. J. Radiat. Oncol., Biol., Phys. 11, 987-999 (1984).

${ }^{8}$ D. D. Leavitt, J. R. Stewart, J. H. Moeller, W. L. Lee, and G. A. Takach, "Electron arc therapy: Design implementation and evaluation of a dynamic multi-vane collimator system,' Int. J. Radiat. Oncol., Biol., Phys. 17, 1080-1094 (1989).

${ }^{9}$ D. D. Leavitt, J. R. Stewart, J. H. Moeller, and L. Earley, “'Optimization of electron arc therapy doses by multi-vane collimator control,' Int. J. Radiat. Oncol., Biol., Phys. 16, 489-496 (1989).

${ }^{10} \mathrm{~A}$. Brahme, "Design principles and clinical possibilities for a new generation of radiation therapy equipment," Acta Oncol. 26, 403-412 (1987).

${ }^{11}$ M. Karlsson, H. Nystrom, and H. Svensson 'Electron beam characteristics of the 50-MeV racetrack microtron,' Med. Phys. 19, 307-315 (1992).

${ }^{12}$ M. Karlsson and B. Zackrisson, "Matching of electron and photon beams with a multi-leaf collimator,"' Radiother. Oncol. 29, 317-326 (1993).

${ }^{13}$ R. van der Laarse, I. A. D. Bruinvis, and M. F. Nooman, "Wall-scattering effects in electron beam collimation," Acta Radiol.: Oncol., Radiat., Phys., Biol. 17, 113-124 (1978).

${ }^{14}$ M. D. Mills, K. R. Hogstrom, and P. R. Almond, "Prediction of electron beam output factors,' Med. Phys. 9, 60-68 (1982).

${ }^{15}$ I. A. D. Bruinvis, A. van Amstel, A. J. Elevelt, and R. van der Laarse, "Calculation of electron beam dose distributions for arbitrarily shaped fields,', Phys. Med. Biol. 28, 667-683 (1983).

${ }^{16}$ I. A. D. Bruinvis and W. A. Mathol, "Calculation of electron beam depth-dose curves and output factors for arbitrary field shapes,' Radiother. Oncol. 11, 395-404 (1988).

${ }^{17}$ B. J. McParland, "A method of calculating the output factors of arbitrarily shaped electron fields,'” Med. Phys. 16, 88-93 (1989).

${ }^{18}$ A. Kassaee, M. D. Altschuler, S. Ayyalsomayajula, and P. Bloch, "Influence of cone design on the electron beam characteristics on clinical accelerators,', Med. Phys. 21, 1671-1676 (1994).
${ }^{19}$ E. E. Klein, Z. Li, and D. Low, "Feasibility study of multileaf collimated electrons with a scattering foil based accelerator,', Radiother. Oncol. 41, 189-196 (1996).

${ }^{20}$ M. E. Masterson, G. S. Mageras, T. LoSasso, E. Joreskog et al., "Preclinical evaluation of the reliability of a $50 \mathrm{MeV}$ racetrack microtron,’'Int. J. Radiat. Oncol., Biol., Phys. 28, 1219-1227 (1994).

${ }^{21}$ M. E. Masterson, C. S. Chui, R. Febo, J. D. Hung et al., "'Beam characteristics of a new generation $50 \mathrm{MeV}$ racetrack microtron,' Med. Phys. 22, 781-792 (1995).

${ }^{22}$ A. Brahme, T. Kraepelien, and H. Svensson, "Electron and photon beams from a $50 \mathrm{MeV}$ racetrack microtron,' Acta Radiol.: Oncol. 19, 305-319 (1980).

${ }^{23}$ F. M. Khan, K. P. Doppke, K. R. Hogstrom, G. J. Kutcher, R. Nath, S.C. Prasad, J. A. Purdy, M. Rozenfeld, and B. L. Werner, 'Clinical electronbeam dosimetry: Report of AAPM RTC Task Group No. 25,' Med. Phys. 18, 73-109 (1991).

${ }^{24}$ K. R. Hogstrom, M. D. Mills, and P. R. Almond, "Electron beam dose calculations,', Phys. Med. Biol. 26, 445-459 (1981).

${ }^{25}$ J. A. Meyer, J. R. Palta, and K. R. Hogstrom, 'Determination of relatively new electron dosimetry measurement techniques on Mevatron 80," Med. Phys. 11, 670-677 (1984).

${ }^{26}$ F. M. Khan, W. Sewchand, and S. H. Leavitt, "Effect of air space on depth dose in electron beam therapy," Radiology 126, 249-251 (1978).

${ }^{27}$ H. Huizenga and P. R. M. Storchi, "The in-air scattering of clinical electron beams as produced by accelerators with scanning beams and diaphragm collimators," Phys. Med. Biol. 32, 1011-1029 (1987).

${ }^{28}$ R. E. George, S. V. Frost, and M. Hartson-Eaton, "Characteristics of electron beams from a medical microtron,' Med. Phys. 13, 533-538 (1986).

${ }^{29}$ P. O'Brien, H. B. Michaels, J. E. Aldrich, and J. W. Andrew, 'Characteristics of electron beams from a new 25-MeV linear accelerator,' Med. Phys. 12, 799-805 (1984).

${ }^{30}$ J. R. Palta, I. K. Daftari, K. M. Ayyangar, and N. Suntharalingam, “'Electron beam characteristics on a Philips SL25,' Med. Phys. 17, 27-34 (1990).

${ }^{31}$ D. M. Roback, F. M. Khan, J. P. Gibbons, and A. Sethi, "Effective SSD for electron beams as a function of energy and beam collimation,', Med. Phys. 22, 2093-2095 (1995).

${ }^{32}$ E. E. Klein, D. A. Low, and J. A. Purdy, "Changes in electron beam dosimetry with a new scattering foil-applicator system on a CL2100C,', Int. J. Radiat. Oncol., Biol., Phys. 32, 483-490 (1995).

${ }^{33}$ A. Brahme, "Geometric properties of clinical electron beams," Acta Radiol., Suppl. 364, 11-19 (1983). 